\title{
The radial velocity acceleration of the 6.7 GHz methanol maser in Mon R2 IRS3
}

\author{
K. Sugiyama ${ }^{1}$, K. Fujisawa ${ }^{2}$, N. Shino ${ }^{1}$, and A. Doi ${ }^{3}$ \\ ${ }^{1}$ Dept. of Physics, Yamaguchi Univ., 1677-1 Yoshida, Yamaguchi, Yamaguchi 753-8512, Japan \\ email: koichiro@yamaguchi-u.ac.jp \\ ${ }^{2}$ The Research Institute of Time Studies, Yamaguchi Univ., 1677-1 Yoshida, Yamaguchi, \\ Yamaguchi 753-8511, Japan \\ ${ }^{3}$ Institute of Space and Astronautical Science, JAXA, Yoshinodai 3-1-1, Chuo-ku, Sagamihara, \\ Kanagawa 252-5210, Japan
}

\begin{abstract}
We present the radial velocity acceleration of the $6.7 \mathrm{GHz}$ methanol maser in a high-mass star-forming region Monoceros R2 (Mon R2). The methanol maser is associated with an infrared source IRS3. The methanol maser of Mon R2 shows at least three spectral features having radial velocities $\left(V_{\mathrm{lsr}}\right)$ of $10.8,12.7$, and $13.2 \mathrm{~km} \mathrm{~s}^{-1}$. The radial velocity of a feature at $V_{\mathrm{lsr}}=12.7 \mathrm{~km} \mathrm{~s}^{-1}$ has changed during ten years from Aug. 1999 to Oct. 2009, corresponding to an acceleration of $0.08 \mathrm{~km} \mathrm{~s}^{-1} \mathrm{yr}^{-1}$. We observed the $6.7 \mathrm{GHz}$ methanol masers of Mon R2 in Oct. 2008 using the Japanese VLBI Network (JVN). Compared with the previous VLBI image obtained in Nov. 1998 using the European VLBI Network (EVN), the maser feature at $V_{\mathrm{lsr}}=12.7 \mathrm{~km} \mathrm{~s}^{-1}$ showed relative proper motions of $\sim 2.5 \mathrm{mas} \mathrm{yr}^{-1}$ (about $10 \mathrm{~km} \mathrm{~s}^{-1}$ at $0.83 \mathrm{kpc}$ ) toward the intensity peak of IRS3. The radial velocity acceleration could be caused by an inflow from a disk or envelope around a high-mass young stellar object (YSO) at IRS3.
\end{abstract}

Keywords. Stars: formation, Masers: methanol, Instrumentation: high angular resolution

\section{Introduction}

Mon R2 is a well-known region consisting of a chain of reflection nebulae, associated with a giant molecular cloud that is one of the closest high-mass star-forming regions to the Sun at a distance of $0.83 \mathrm{kpc}$ (Racine 1968; see the review by Carpenter \& Hodapp 2008). There are several near infrared sources identified on the K-band images (Carpenter et al. 1997), and the brightest source was labeled as IRS3. The IRS3 source showed a flattened spatial structure in polarimetry observations at position angle $\sim 140^{\circ}$, which was interpreted as a circumstellar disk around a high-mass YSO (Yao et al. 1997). Later high spatial-resolution observations at near infrared bands showed three separated YSOs in the IRS3 field, and each YSO shows micro-jets in the northeast-southwest directions.

The methanol maser at $6.7 \mathrm{GHz}$ in Mon R2 is associated with IRS3, and each maser spot was found to be linearly distributed in the northeast-southwest direction in previous VLBI observations conducted in Nov. 1998 (Minier et al. 2000). We observed the $6.7 \mathrm{GHz}$ methanol masers in Mon R2 every year from 2004 to 2009 using the Yamaguchi 32-m radio telescope and in Oct. 2008 using the JVN.

\section{Radial Velocity Acceleration and Relative Proper Motions}

We conducted the $6.7 \mathrm{GHz}$ methanol maser observations every year from 2004 to 2009 using the Yamaguchi 32-m, and compared with the data obtained in 1999 using the Torun 32-m radio telescope (Szymczak et al. 2000). The methanol maser of Mon R2 shows at least three spectral features having radial velocities $\left(V_{\mathrm{lsr}}\right)$ of $10.8,12.7$, and $13.2 \mathrm{~km} \mathrm{~s}^{-1}$ 

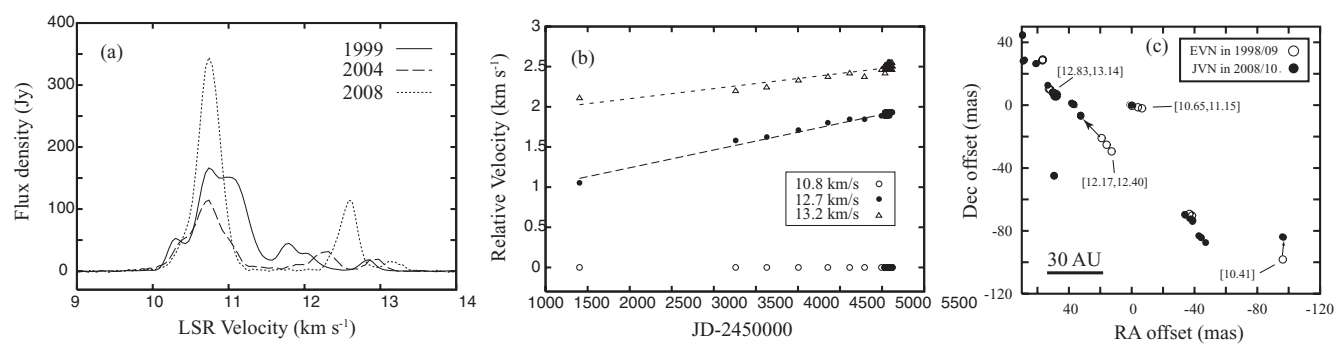

Figure 1. The $6.7 \mathrm{GHz}$ methanol maser in Mon R2. (a) Spectra in 1999 with the Torun 32-m (solid line) and in 2004, 2008 with the Yamaguchi 32-m (dashed and dotted lines). (b) Radial velocity acceleration relative to the feature at $V_{\mathrm{lsr}}=10.8 \mathrm{~km} \mathrm{~s}^{-1}$. Each line shows the accelerations obtained from least-squares fits. (c) Overlaid VLBI images in Nov. 1998 (Minier et al. 2000, open circle) and Oct. 2008 (our work, filled circle). Attached numbers indicate a range of radial velocities for each spot, and arrows show proper motions relative to the reference spot at $V_{\mathrm{lsr}}=10.8 \mathrm{~km} \mathrm{~s}^{-1}$.

in 2008. Uncertainties of absolute radial velocities in both observations, unfortunately, were large of $\sim 0.3-0.4 \mathrm{~km} \mathrm{~s}^{-1}$. We compared radial velocities, therefore, relative to the strong maser feature at $V_{\mathrm{lsr}}=10.8 \mathrm{~km} \mathrm{~s}^{-1}$. As shown in Fig. 1(a), the spectral feature at $V_{\mathrm{lsr}}=11.8$ and $12.1 \mathrm{~km} \mathrm{~s}^{-1}$ in 1999 merged to one spectral feature, and changed to the red-shifted direction indicating a radial velocity of $12.3,12.7 \mathrm{~km} \mathrm{~s}^{-1}$ in 2004 and 2008, respectively. The spectral feature at $V_{\mathrm{lsr}}=12.9 \mathrm{~km} \mathrm{~s}^{-1}$ in 1999 also changed to the red-shifted direction, but its variation was smaller than that of the former. Each acceleration was estimated as 0.08 and $0.05 \mathrm{~km} \mathrm{~s}^{-1} \mathrm{yr}^{-1}$ by a least-squares fit (Fig. 1b).

We overlaid our VLBI image obtained using the JVN in Oct. 2008 on the previous VLBI image obtained using the EVN in Nov. 1998 (Minier et al. 2000) using as an origin the reference maser spot at $V_{\mathrm{lsr}}=10.8 \mathrm{~km} \mathrm{~s}^{-1}$ (Fig. 1c). On the basis of the overlaid VLBI images, the acceleration of maser spots at $V_{\mathrm{lsr}}=12.7 \mathrm{~km} \mathrm{~s}^{-1}$ moved toward the northeast direction, which was parallel to the direction of the micro-jets. The absolute positional accuracy of the high spatial-resolution map at near infrared bands, unfortunately, was $\sim 1$ arcsec, which was not enough to understand which YSOs were suitable as an exciting source of the methanol masers. We compared our data with the flattened spatial structure obtained from polarimetry observations and therefore interpreted it as due to a circumstellar disk. The intensity peak of IRS3 in the disk, which could correspond to a position of the exciting YSO, is located at the northeast direction from a cluster of the methanol maser spots. Given the relative proper motions of the methanol maser toward the central exciting YSO obtained in our work, the radial velocity acceleration could be caused by an inflow from a disk or envelope around a high-mass YSO at IRS3.

In order to correctly understand the causes of the radial velocity acceleration, we will measure the absolute proper motions of the methanol masers in Mon R2, and obtain high spatial-resolution maps at near infrared bands with high positional accuracies.

\section{References}

Carpenter, J. M. \& Hodapp, K. W. 2008, Handbook of Star Forming Regions, Volume I, 899

Carpenter, J. M., Meyer, M. R., Dougados, C., et al. 1997, AJ, 114, 198

Minier, V., Booth, R. S., \& Conway, J. E. 2000, A\& A, 362, 1093

Racine, R. 1968, AJ, 73, 233

Szymczak, M., Hrynek, G., \& Kus, A. J. 2000, A\&AS, 143, 2

Yao, Y., Hirata, N., Ishii, M., et al. 1997, ApJ, 490, 281 